Voix et Images

\title{
Le concept d'adolescence : évolution et représentation dans la littérature québécoise pour la jeunesse
}

\section{Françoise Lepage}

Volume 25, numéro 2 (74), hiver 2000

Le champ littéraire de la jeunesse au carrefour de la recherche universitaire

URI : https://id.erudit.org/iderudit/201478ar

DOI : https://doi.org/10.7202/201478ar

Aller au sommaire du numéro

\section{Éditeur(s)}

Université du Québec à Montréal

\section{ISSN}

0318-9201 (imprimé)

1705-933X (numérique)

Découvrir la revue

Citer cet article

Lepage, F. (2000). Le concept d'adolescence : évolution et représentation dans la littérature québécoise pour la jeunesse. Voix et Images, 25(2), 240-250.

https://doi.org/10.7202/201478ar d'utilisation que vous pouvez consulter en ligne. 


\title{
Le concept d'adolescence : évolution et représentation dans la littérature québécoise pour la jeunesse
}

\author{
Françoise Lepage, Université d'Ottawa
}

\begin{abstract}
Contrairement à la littérature pour adultes qui présente l'enfance où l'adolescence telles que les adultes se les représentent, la littérature pour la jeunesse constitue un corpus de choix pour suivre l'évolution du concept d'adolescence. Bien que le terme existe depuis fort longtemps, l'adolescence n'est reconnue comme groupe social particulier que depuis le $x^{e}$ siècle. La crise économique des années trente, le nouvel ordre social de l'après-guerre, les manifestations des années soixante, sont autant de remises en question qui ont permis aux adolescents de se constituer en groupe social disposant d'une culture distincte. L'évolution du concept d'adolescence est à l'origine de modifications considérables dans la thématique des livres destinès à cet âge. En établissant un parallèle entre l'bistoire du concept et le contenu de la littérature pour adolescents, le présent article permet de comprendre les plus récents aspects de cette littérature.
\end{abstract}

De nos jours, il ne viendrait à l'esprit de personne de contester l'existence et la spécificité de ces quelques années de la vie qui marquent le passage de l'enfance à l'âge adulte et que l'on appelle l'adolescence. Lès adolescents constituent aujourd'hui un groupe social à part entière, ayant ses propres modes, ses propres valeurs et, surtout, sa propre culture. Les caractéristiques de l'adolescence et les conséquences qui en découlent font tellement partie de notre paysage social et culturel que cette étape de la vie humaine nous paraît être une réalité universelle qui transcende l'espace et le tèmps. Or, il n'en est rien. Il s'agit d'un concept qui a beaucoup évolué au cours des siècles et qui ne s'est véritablement épanoui que dans la deuxième moitié du $\mathrm{xx}^{\mathrm{e}}$ siècle; donc tout récemment. Le présent article se propose non pas d'en retracer l'évolution, travail qui a déjà été fait maintes fois ${ }^{1}$ — je me contenterai d'en évoquer les très

1. Voir en particulier Rolf E. Muuss, Theories of Adolescence, New York, McGraw Hill, 1962, 352 p., ainsi que Encyclopedia of Adolescence, Richard M. Lerner, Anne C. Petersen 
grandes lignes - , mais d'en montrer le développement tel qu'il se manifeste dans la littérature pour la jeunesse québécoise essentiellement, bien qu'à titre comparatif un exemple sera emprunté à la littérature française.

\section{Évolution du concept d'adolescence}

On parle déjà d'adolescence dans les saintes Écritures, dans l'Ecclésiaste en particulier, avec le sens très général de jeunesse. Il en est aussi question chez Platon et Aristote, celui-ci proposant un découpage de la jeunesse en trois périodes de sept ans, sans toutefois employer le terme d'adolescence ${ }^{2}$. En fait, c'est sous l'influence des nécessités de l'enseignement que vont se préciser les différentes étapes de la jeunesse. L'humaniste et pédagogue tchèque, Ian Amos Comenius (1592-1670), propose d'adapter l'enseignement aux capacités de chaque catégorie d'âge, qu'il divise en trois périodes de six ans. Dans ses grandes lignes, ce schéma sert encore de nos jours. La division des âges étant liée à l'instruction, on constate, au fil de l'histoire, une différence entre les classes supérieures de la société et les classes populaires. Les fils de la bourgeoisie qui allaient à l'école pouvaient vivre un simulacre d'adolescence pendant la période où ils étaient coupés de leur famille et donc de la société adulte. La vie de collège leur permettait de se regrouper, d'avoir des loisirs et de partager des goûts communs. Il ne faudrait cependant pas conclure que le concept d'adolescence a pris naissance aux XVIII ${ }^{\mathrm{e}}$ et $\mathrm{XIX}^{\mathrm{e}}$ siècle, car de ces jeunes qui fréquentaient les collèges on attendait qu'ils se comportent comme des adultes. Leurs lectures, leurs goûts devaient être conformes à ceux de leurs prédécesseurs. L'enfance et la jeunesse n'ayant ni l'importance ni l'attrait qu'on leur reconnaît aujourd'hui, il fallait en sortir au plus vite pour devenir une personne digne d'intérêt, c'est-à-dire un adulte. Les éducateurs se chargeaient de leur faire brûler les étapes en imposant la voie à suivre. Il n'y avait pas de place pour la dissidence, ni même pour l'individualité. Quant aux jeunes des classes populaires, peu ou pas scolarisés, ils étaient immédiatement absorbés par la société adulte au sortir de l'enfance, comme travailleurs agricoles, bûcherons, apprentis ou manouvres. L'adolescence n'existait pas dans les faits.

\section{Adolescence et littérature pour la jeunesse}

Cette situation, qui se perpétue immuablement depuis des siècles, commence à se miner de l'intérieur après 1920. Les progrès techniques éliminent de nombreux postes dans les usines, et certaines personnes s'élèvent contre l'exploitation dont les enfants sont victimes dans les

et Joanne Brooks-Gunn (dir.), New York, Garland Publishing, 1991, 2 vol., encyclopédie à laquelle nous empruntons les grandes lignes de l'évolution du concept.

2. Aristote parle plutôt de petite enfance (0-7 ans), d'enfance (8-14 ans) et de jeunesse (15-21 ans). 
manufactures. Les jeunes ne sont plus aussi bienvenus sur le marché du travail et ils doivent être protégés. Cette nouvelle conjoncture conduit très lentement à un changement dans les mentalités. C'est dans ce contexte que s'inscrit la fondation de L'Oiseau bleu (1921-1940), première revue québécoise pour la jeunesse, créée dans le but de fournir des lectures et des loisirs sains à une population de plus en plus urbanisée. Pour les éducateurs sensibles à l'idéologie agriculturiste de Lionel Groulx, les jeunes sont exposés, en milieu urbain, à de multiples dangers, dont l'influence des illustrés américains n'est pas le moindre. De façon très symptomatique, la revue s'annonce comme étant destinée aux lecteurs de trois à dix-huit ans. En dehors du fait que ce type de publicité constitue un poncif à l'époque, il est clair que les instigateurs dè la revue ne se sont pas interrogés sur une éventuelle spécificité des âges.

Si l'on examine les premières cuvres québécoises, écrites à partir des années vingt, cette hypothèse se confirme. L'action de la célèbre série des Aventures de Perrine et Charlot de Marie-Claire Daveluy se déroule de 1636 à 1660, soit sur une période de vingt-quatre ans. Au début du roman, Perrine et Charlot ont respectivement huit et six ans; dans le sixième et dernier volume, ils sont âgés de trente-deux et trente ans. En fait, seuls les deux premiers volumes mettent en scène des enfants; ensuite, ce sont de grands adolescents, puis des adultes. Avec le sixième volume, intitulé Le coeur de Perrine, où il est question de fiançailles, on entre carrément dans le roman pour adolescentes. Au début, la série semble destinée aux enfants, tandis qu'à la fin, elle traite de problèmes d'adolescence. Une telle évolution chez les personnages se fait plus rare dans la littérature actuelle pour la jeunesse, où le temps du récit tend à se réduire à quelques mois, tout au plus à un maximum de deux ou trois ans. Dans certaines séries, en particulier celles destinées aux lecteurs de huit à dix ans, il y a un grand décalage entre le temps de l'écriture et le temps du récit; cela évite d'avoir à faire vieillir le héros ou l'hérö̈ne entre chacune de leurs aventures, tout en permettant de mieux respecter la spécificité des lecteurs selon leur âge. Cette différence dans le traitement du temps souligne aussi la transformation des valeurs exprimées dans les œuvres romanesques. Marie-Claire Daveluy se propose avant tout de faire connaître l'histoire de la Nouvelle-France à l'époque des guerres iroquoises; l'âge des personnages et, par ricochet, celui des lecteurs, importent donc peu. Son propos s'adresse à l'ensemble de la collectivité, ce qui s'oppose à l'atmosphère intimiste et individualiste des ouvres contemporaines où les personnages sont conçus comme des doubles du lecteur. Cette imprécision concernant l'âge des personnages qu'on remarque dans la série Perrine et Charlot se retrouve dans Le Richelieu hérö̈que (1940). Qui sont les personnages principaux? Sont-ce Mathilde et Olivier, déjà de jeunes adultes, ou Josephte et Michel qui sont des enfants? Manifestement, la question ne se posait pas à l'époque. 
Plus passionnant encore pour cerner l'évolution du concept d'adolescence au Québec est le roman du père Paul-Émile Farley, Jean-Paul, écrit en 1929, à l'intention des élèves des collèges. Clerc de Saint-Viateur, professeur au séminaire de Joliette jusqu'en 1934, le père Farley s'intéressait à l'adolescence, comme le prouve une brochure publiée en 1927, donc antérieure au roman, et qui s'intitulait Le caractère de l'adolescent ${ }^{3}$. Cet essai contient nombre de remarques très pertinentes, mais les conclusions qu'en tire le pédagogue (et que son roman servira à illustrer) s'avèrent pour le moins décevantes. S'il reconnaît la spécificité de cette époque de la vie, c'est aussitôt pour la nier, car "éduquer un jeune homme, c'est le mettre dans un moule et parvenir à incorporer le moule à sa personne. Le jour où la discipline sera devenue chez lui une habitude et une conviction, son éducation sera faite ${ }^{4}$.

Didactique et anti-romanesque au plus haut point, Jean-Paul constitue toutefois une ouvre révélatrice à la fois des méthodes d'éducation et de la valeur que l'on accordait à l'adolescence à l'aube des années trente. Le personnage éponyme a dix-sept ans et il étudie dans un collège classique. Au cours des vacances d'été, il fait fortuitement la connaissance d'une jeune fille qui ne le laisse pas indifférent. Pensionnaire et ne pouvant recevoir de lettres d'amour au collège, il s'arrange avec un externe qui se charge de faire le facteur. Du moins, c'est ce que ce camarade lui dit. En fait, ce mauvais apôtre fait écrire des lettres par sa propre sœur, laquelle invente des anniversaires dans le but de s'approprier les boîtes de chocolat que le pauvre Jean-Paul croit envoyer à sa bien-aimée. Le narrateur, évidemment hétérodiégétique, s'arrange, on le voit, pour ridiculiser l'adolescent par le biais de ses amours. Il banalise les sentiments du jeune homme, les réduisant à la simple satisfaction d'un ego boursouflé. Cette relation amoureuse est, selon le narrateur, "affaire de vanité peut-être plus encore que d'amour. Devant ses intimes, cela ne le poserait-il pas en héros de roman ou tout au moins de romance ${ }^{5}$ ? $\mathrm{Le}$ confesseur de Jean-Paul lui révèle toute l'étendue de la supercherie. Ainsi humilié et confus, se croyant la risée de tous ses camarades, isolé, JeanPaul est fin prêt pour passer dans le fameux moule qui fait les bonnes éducations. Dirigé par son confesseur, qui tire toutes les ficelles dans. le dos de l'adolescent, l'ardent Jean-Paul du début, lecteur du Lac de Lamartine et de Tristesse d'Olympio de Victor Hugo, finit au Postulat des Pères blancs de Québec, où il se prépare à devenir missionnaire.

Ce roman emblématique, révélateur de l'opinion de l'élite québécoise face à l'adolescence, fait de cette période de la vie un moment d'errance

3. Paul-Émile Farley, c.s.v., Le caractère de l'adolescent, Montréal, L'École sociale populaire, coll. "L’École sociale populaire. Publication mensuelle", $\mathrm{n}^{\circ} 158,1927,32$ p.

4. Ibid., p. 24 .

5. Id., Jean-Paul, Montréal, Les clercs de Saint-Viateur, 1929, p. 69. 
des sentiments et des idées, et plus vite cet égarement sera réprimé mieux cela vaudra. Il faut dire que l'état d'esprit qui caractérise l'adolescence est tout à fait contraire à la doctrine chrétienne et, par conséquent, ce stade de l'évolution individuelle ne pouvait être prisé. L'adolescent est replié sur lui-même, alors que l'Église prêche l'ouverture aux autres; il ne domine pas très bien ses pulsions, il est avide de faire des expériences, ce qui est contraire à l'exigence de pureté préconisée par l'enseignement chrétien.

On pourrait se demander si cette conception de l'adolescence était universelle ou si elle relevait plutôt de l'idéologie religieuse transmise aux élèves de collèges catholiques dans une société fermée sur elle-même. La comparaison avec un célèbre roman français permettra d'étayer cette hypothèse.

Le grand Meaulnes d'Alain-Fournier paraît pour la première fois de juillet à novembre 1913 dans La Nouvelle Revue française, soit seize ans avant Jean-Paul. Dans ce roman, l'adolescence se révèle être une période d'inconfort, de malaise. Bien qu'à la fin Augustin Meaulnes soit père de famille, il est clair qu'il n'accepte pas son statut d'adulte, contraire pour lui à son idéal d'innocence et de pureté. Le narrateur, François Seurel, nous le montre avec sa petite fille dans les bras et ajoute: "Déjà je l'imaginais, la nuit, enveloppant sa fille dans un manteau, et partant avec elle pour de nouvelles aventures". C'est la dernière phrase du livre. Par l'intermédiaire de sa fille, Meaulnes renoue avec le monde de l'enfance. Il se tient constamment sur le fil du rasoir entre l'enfance et l'âge adulte, entre le domaine enchanté, monde de la fantaisie, du rêve et de l'amour pur, et l'appel du bohémien, Frantz de Galais, qui le force à faire face aux aspects dramatiques de la vie (tentative de suicide, compromis, trahison, amours coupables). Meaulnes n'est pas capable de passer à l'âge adulte et il reste pris dans le malaise de l'adolescence. À l'époque de la publication du roman, l'adolescence est perçue comme un très court moment de l'existence, un passage. Le roman d'Alain-Fournier, et c'est ce qui fait sa nouveauté, va amener la reconnaissance de cette période trouble de la vie et va tendre à la mythifier. Pour François Seurel, double du lecteur, Meaulnes représente la fascination de l'adolescence, d'un monde tourmenté et transitoire. Contrairement à ce qui se passe dans le roman du père Farley, l'adolescence est exaltée, magnifiée dans l'œuvre du romancier français.

\section{Le tournant de la Dépression et de la crise économique}

Le point tournant de l'évolution du concept d'adolescence apparaît au moment de la Dépression. Les conditions de vie de la jeunesse changent. Le travail est rare et ce sont les derniers arrivés sur le marché du travail qui font les premiers l'expérience du chômage. Même les jeunes instruits 
ne trouvent pas d'emploi. En juin 1935, Jean-Charles Harvey prononçe une conférence à Québec devant les membres du club Kiwanis ${ }^{6}$ pour éveiller l'opinion publique sur la situation critique dans laquelle se trouve la jeunesse:

Dans les circonstances, je ne suis pas surpris de voir tant d'impatience chez les jeunes. Dans nos grands centres se dessinent divers mouvements presque silencieux, mais assez violents, où perce un désir de se grouper, de déclencher, soit par la parole, soit par l'écrit, une action à la fois politique et sociale à l'aide de laquelle on espère emporter les places d'assaut. [...] Ce qui se prépare dans nos agglomérations urbaines, ce n'est pas seulement la haine contre un état de choses ou contre des lois, mais aussi la rupture entre deux générations ${ }^{7}$.

Et, plus loin, il parle de "l'abîme que six années de dépression économique ont creusé entre deux générations voisines ${ }^{8}$. Cette période provoque un profond changement social d'où naîtra l'adolescence comme groupe social. Car que font ces jeunes qui ne trouvent plus à s'employer dans la société des adultes, qui autrefois les absorbait au sortir de l'enfance? Ils continuent à aller à l'école et s'inscrivent en masse dans les établissements d'enseignement secondaire. Ainsi coupés du monde adulte, ils prennent conscience de leur singularité en tant que groupe et c'est à partir de cette époque, ou plus exactement après la guerre - car la Seconde Guerre mondiale a momentanément rouvert les portes des manufactures aux adolescents -, qu'a commencé à apparaître une culture de l'adolescence. Au tout début des années cinquante se répand partout en Occident la notion de "teenagers". Des films comme La fureur de vivre (1955), avec James Dean dans le rôle d'un adolescent anticonformiste et rebelle, peignent une certaine adolescence, qui inquiète les adultes, et fournissent de nouveaux héros aux jeunes. Durant ces années commencent également à fleurir les modes vestimentaires adolescentes (surtout pour les filles), et l'emploi d'un langage de caste. À la fin de la décennie, le rock'n'roll exploite de nouveaux thèmes: l'amour, le sexe, l'opposition aux adultes 9 .

Dans la littérature québécoise pour la jeunesse, on tient de plus en plus compte de tranches d'âge précises.. Des auteurs comme Tavi (pseudonyme d'Albert Tessier) ou Jeanne L'Archevêque-Duguay s'adressent très clairement aux adolescentes dans des ouvrages comme Femmes de maison dépareillées (1942) ou Pleine floraison (1946). De nombreux

6. Conférence publiée la même année sous le titre Jeunesse (Montréal, Éditions de Vivre, coll. "Les cahiers noirs", 1935, 59 p.).

7. Ibid., p. 21.

8. Ibid., p. 29

9. Ce panorama de la culture adolescente dans les années cinquante est tiré de Jeffrey E. Mirel, "Twentieth-Century America, Adolescence in", Encyclopedia of Adolescence, vol. II, p. 1161. 
romans voient le jour à la fin des années quarante et durant les années cinquante et traitent, selon la morale chrétienne, des problèmes des adolescentes. Je ne citerai, pour exemples, que Geneviève de Francheville ( $L e$ calvaire de Monique [1953]; La sacrifiée [1959]), et Reine Malouin (Profonds destins [1957]; J'ai choisi le malbeur [1958]). On voit même apparaître, au début des années soixante, des guides à l'usage des adolescentes: le très conservateur Écoute, ma mie (1961) de Tante Chantal (pseudonyme de Cécile Lachaîne-Brosseau) et Maman, dis-moi coimment être jolie (1961) de Janette Bertrand, beaucoup plus proche de la grande préoccupation des filles à l'aube de l'adolescence: la séduction. Quant aux garçons, on leur destine des romans d'aventures qui mettent en scène des héros de leur âge, comme Prisonniers des cavernes de Guy Boulizon (1950) ou Le prisonnier du vieux manoir d'Ambroise Lafortune (1952).

Si la notion de public cible se précise, les ouvrages ne donnent pas encore la parole aux adolescents. On ne cherche pas à exprimer leur point de vue, mais plutôt à leur indiquer la voie à suivre. Le cabochon d'André Major, sous-titré "Roman pour adolescents" et publié en 1964, constitue un exemple représentatif. André Major destinait son roman à Vie étudiante, journal de la JEC, mais la publication dut être interrompue à cause de l'antagonisme qui s'était élevé entre les autorités et la presse étudiante jugée trop libre. Le cabochon a servi de bouc émissaire à un règlement de compte entre "les Vieillards", comme les appelle Major, et les journaux étudiants. Ce roman ne contient cependant rien de scabreux si ce n'est, et c'est déjà beaucoup trop pour l'époque, qu'Antoine, le personnage principal, est un "décrocheur". Il abandonne l'école et veut trouver sa propre voie. Cette indépendance de caractère ne pouvait plaire. L'individualisme de la jeunesse, son esprit frondeur, sont encore considérés comme des comportements indésirables. La seule façon de contourner l'autorité est de publier en dehors des organismes qu'elle contrôle, soit aux Éditions Parti pris dans le cas d'André Major. À la fin des années cinquante et au début des années soixante, Paule Daveluy a la chance de tomber sur un éditeur plus large d'esprit, qui accepte son premier roman pour adolescentes, audacieux pour l'époque, L'été enchanté (1958) ${ }^{10}$. Rosanne, personnage principal, part, l'été de ses seize ans, à la conquête du jeune médecin nouvellement installé dans la petite ville où elle passe ses vacances. Exceptionnelle sur toute la ligne, la série Rosanne ose pour la première fois peindre le monde tel que le voit une adolescente et permet une incursion dans la psychologie de son héroïne $^{11}$. Le choix d'un narrateur homodiégétique témoigne de cette

10. Il s'agit des Éditions de l'Atelier (1958), qui relevaient des clercs de Sainte-Croix.

11. Cette série comportait à l'origine trois romans. À L'été enchanté faisaient suite Drôle d'automne (1961) et Cet hiver-là (1967). Cher printemps, dernier volume de la série, n'a été publié, sous une forme condensée, qu'en 1977 (Rosanne et la vie, Fides, coll. "Goéland ") et, dans sa version intégrale, qu'en 1996, aux éditions Québec Amérique. 
avancée de la parole adolescente. Paule Daveluy poursuit dans cette tonalité plus moderne avec ses autres œuvres, en particulier avec la série Sylvette (Sylvette et les adultes [1962] et Sylvette sous la tente bleue [1964]). Cette voie nouvelle ne sera plus empruntée avant les années quatre-vingt.

\section{Les mouvements étudiants dans les années soixante}

Un nouveau pas va être franchi à la fin des années soixante et au début des années soixante-dix, étape décisive qui va donner à la littérature pour adolescents le visage qu'on lui connaît aujourd'hui. On doit ces transformations aux révoltes étudiantes de la fin de la décennie d'une part et, d'autre part, à l'influence d'œuvres étrangères comme celle de l'Américaine Judy Blume. Les manifestations de la jeunesse à la fin des années soixante vont remettre en question les structures hiérarchiques de la société, jusque dans sa cellule de base qu'est la famille, tout en profitant aussi aux femmes et aux jeunes filles qui vont se libérer des carcans et des tabous entourant leur sexualité. Simultanément, la recherche scientifique sur l'adolescence s'intensifie ${ }^{12}$, ses résultats se répandent et imprègnent les œuvres créées pour la jeunesse. Ainsi en est-il du concept de "déidéalisation" par lequel Peter Blos explique certains états psychiques de l'adolescence:

Par ce processus, la jeune personne défait les images parentales idéales (père et mère) de son enfance en y découvrant toutes les imperfections; elle défait aussi l'idéal de soi qu'elle s'était fait en découvrant ses limites personnelles. [...] Le tumulte adolescent est l'effet normal de la croissance et témoigne de la reconstruction d'une nouvelle personne où les objets d'amour de l'enfance perdent leur perfection imaginée et sont réintégrés avec leurs bons côtés et leurs mauvais côtés dans une mosaïque différenciée et autonome ${ }^{13}$.

Remise en question de l'autorité, déidéalisation des images parentales: on a là toute la différence entre L'été enchanté (1958) de Paule Daveluy et Cassiopée ou l'été polonais (1988) de Michèle Marineau. Entre ces deux romans, trente ans où s'inscrit une affirmation croissante de la psychologie adolescente.

Parallèlement, la romancière américaine Judy Blume commence à publier des romans qui auront un grand retentissement dans le public lecteur. Avec Dieu, tu es là, c'est moi Margaret, publié en 1970, la romancière américaine inaugure la vogue des romans réalistes, qui misent sur la

12. Anne C. Petersen ("History of Research on Adolescence", Encyclopedia of Adolescence, vol. I, p. 499) signale qu'à la fin des années soixante et au début des années soixantedix s'est manifesté, dans la société, un très grand intérêt pour l'adolescence. Elle ajoute que cet intérêt a contribué à stimuler la recherche sur cette période de la vie.

13. Peter Blos, The Adolescent Passage: Developmental Issues, New York, International University Press, 1979, cité par Paul Cloutier, Psychologie de l'adolescence, Chicoutimi, Gaëtan Morin éditeur, 1982, p. 14. 
sincérité du narrateur induite par le ton du journal intime et privilégient le dialogue comme technique narrative. L'inquiétude que suscitent l'apparition des premières menstruations, les relations avec le sexe opposé, la première expérience sexuelle (Forever, 1975), tous ces sujets dont on ne parlait pas auparavant font leur entrée dans le roman pour la jeunesse. Pendant que la critique accueille ces nouveautés avec de grandes réserves, les jeunes s'arrachent ces romans qui se vendent à plus de vingtcinq millions d'exemplaires ${ }^{14}$. Pendant ce temps, au Québec, on tente de plus en plus de donner la parole aux adolescents, comme en témoigne la collection "L'Actuelle/Jeunesse", qui paraît aux éditions de L'Actuelle de 1971 à 1975. Cette maison d'édition organisait un concours à l'intention des adolescents et publiait les meilleurs manuscrits. Dix courts romans ont ainsi vu le jour, assez conservateurs pour la plupart, si l'on excepte les quatre romans de science-fiction, genre qui, en lui-même; fait alors figure de nouveauté dans la littérature pour la jeunesse ${ }^{15}$. Seul L'engrenage (1973) de Claudine Numainville évoque la réalité de certains jeunes de l'époque: la drogue, la solitude, l'indifférence du père, la révolte contre la société, la vie dans une commune. Les réticences des adultes à l'égard du roman réaliste ont retardé l'expansion de ce type d'œuvres, et il faudra attendre, au Québec et dans d'autres pays occidentaux, le milieu des années quatre-vingt pour en voir l'apparition. À ce moment-là, l'adolescence s'est définitivement imposée dans toute sa spécificité.

\section{Littérature pour adolescents et critique universitaire}

On relève également des traces de ces changements et de ces réticences dans la critique universitaire. On a vu que les romans de Judy Blume avaient été accueillis avec réserve par les adultes et, dans les années quatre-vingt, la romancière n'avait encore reçu aucun prix littéraire important ${ }^{16}$. De l'autre côté de l'Atlantique, lorsque Marc Soriano publie sa première étude sur le livre pour la jeunesse en 1959, il l'intitule Guide de la littérature enfantine. En 1975, le même ouvrage remanié et augmenté devient Guide de littérature pour la jeunesse, modification qui dénote une volonté d'élargir le public. On est assez surpris de constater que ce guide ne comporte aucune entrée à̀ "adolescence", mais on comprend très vite, lorsqu'on prend connaissance de l'article consacré aux classes d'âge, qu'en 1975, les adultes n'étaient pas encore prêts à remettre en question les tabous. Marc Soriano remarque que les adolescents forment un public numériquement important, que les éditeurs s'efforcent de conquérir, mais il ajoute:

14. Faith McNulty, "L'éducation sentimentale selon Judy Blume", La Revne des livres pour enfants, $\mathrm{n}^{\circ} 102$, mai-juin 1985 , p. 36.

15. Au Québec, on doit le premier roman de science-fiction pour la jeunessè à Guy Bouchard qui publie Vénus via Atlantide en 1961.

16. Faith McNulty, loc. cit., p. 36. 
Le paradoxe, dans nos sociétés libérales, c'est que cette conquête est pratiquement impossible. En effet, pour intéresser ce type de public, il faudrait utiliser franchement ses motivations réelles: la sexualité, la vie affective, l'actualité avec sẹ problèmes économiques, politiques et sociaux. Or une loi non écrite, mais impérative de notre société veut qu'on évite [...] d'aborder devant les jeunes ces sujets litigieux ${ }^{17}$.

Cette analyse est à la fois clairvoyante dans l'expression de la recette à suivre et très conservatrice dans la vision de l'avenir.

Pourtant, des romans comme Le dernier des raisins de Raymond Plante (Québec Amérique, 1986) ou Cassiopée ou l'été polonais de Michèle Marineau (Québec Amérique, 1988) sont en passe de devenir de nouveaux classiques. Le narrateur, double du lecteur, partage avec celui-ci ses interrogations, les incidents de son quotidien, sa conception de la vie, ses premiers émois amoureux. Les problèmes abordés (éveil de la sexualité, relations avec les parents, amitiés, jalousie et vie scolaire) concernent tous les adolescents, même si le contexte urbain, le milieu bourgeois ou petitbourgeois, les problèmes des familles reconstituées, ne correspondent pas nécessairement au cadre de vie de tous les jeunes d'aujourd'hui. Outre une thématique qui reflète étroitement les préoccupations adolescentes, un habile dosage d'humour et d'émotion donne à ces ouvrages de bonnes chances de transcender l'espace et le temps. D'autres auteurs, comme Anique Poitras, Dominique Demers ou Marie-Francine Hébert, réussissent aussi à captiver les jeunes lecteurs, même si leurs œuvres baignent dans des tonalités plus sombres. Cette littérature, hier impensable, existe bel et bien aujourd'hui et aborde un vaste éventail de ces "sujets litigieux". À la découverte de la sexualité s'ajoutent les conflits parents-enfants, la drogue, les grossesses d'adolescentes, les fugues, le suicide, les jeunes sans-abri, la violence.

La notion d'adolescence, nous venons de le voir, a beaucoup évolué en un siècle et cette évolution n'est peut-être pas terminée. L'âge de l'entrée dans l'adolescence, que symbolise, pour les filles, l'apparition des premières menstruations, n'a cessé de s'abaisser depuis un siècle et demi, passant de seize ans en 1850 à douze ans aujourd'hui. De nos jours, "plus d'un quart des fillettes se changent en jeunes filles dès 10 ou 11 ans ${ }^{18}$. Cette précocité de la maturité sexuelle permet de déduire que les collections actuelles, dites "pour adolescents", sont lues par des lecteurs et des lectrices beaucoup plus jeunes que prévu. Un nouveau vide se crée alors entre les livres pour adolescents, qui auront été lus entre dix et treize ans environ, et la production pour adultes. Certaines maisons d'édition comme Héritage structurent depuis longtemps leurs collections pour adolescents - la collection "Échos", par exemple - en trois

17. Marc Soriano, Guide de littérature pour la jeunesse, Paris, Flammarion, 1975, p. 120.

18. Marion Festraëts, "Génération Lolita", L'Express, 18 février 1999, p. 26-27. 
niveaux: douze ans et plus; quatorze ans et plus; et jeunes adultes. On voit donc apparaitre un nouveau concept, celui des "jeunes adultes", né aux États-Unis dans la deuxième moitié des années quatre-vingt, qui, selon un critique français, produit "une littérature de second ordre" usant des "grossières ficelles des paralittératures adultes non encore légitimées (romans sentimentaux, heroïc-fantasy, horreur). Les structures du récit et les valeurs véhiculées restent très traditionnelles ${ }^{19}$ ". En lançant leur collection "Roman 16/96" en 1995, les Éditions de La courte échelle cherchent, entre autres, à rejoindre les jeunes qui ont passé l'âge des collections pour la jeunesse. Mais à la différence des productions américaines qui offrent une sous-littérature, les romans de la collection "16/96" sont de véritables œuvres littéraires produites par des auteurs reconnus, comme Marie-Danielle Croteau, Stanley Péan ou Raymond Plante.

Cette étude nous a permis de suivre l'évolution du concept d'adolescence et ses répercussions dans la littérature pour la jeunesse. Confondus à l'origine avec les adultes, les adolescents n'avaient pas d'existence véritable. L'adolescence n'était qu'un moment que les éducateurs s'efforçaient d'écourter au maximum. Ce n'est qu'au $x^{\mathrm{e}}$ siècle que les psychologues commencent à s'intéresser à cet âge de la vie et c'est à la suite d'une série de secousses sociales qu'elle s'impose comme groupe. La Dépression est déterminante dans la mesure où elle creuse un fossé entre les générations, regroupe les jeunes dans les écoles secondaires et fait naître une culture propre à l'adolescence. Les mouvements étudiants dans les années soixante et la remise en question de l'autorité, la libéralisation des mœurs qui s'ensuivit, l'influence de romancières étrangères, le développement de la recherche sur l'adolescence, tous ces phénomènes vont finir par balayer les dernières réticences. Le roman pour adolescents aborde enfin les aspects de la vie qui préoccupent ses lecteurs : les tabous d'hier nourrissent les œuvres d'aujourd'hui. Une imposante littérature est née, objet de consommation qui forme, informe et divertit les adolescents d'aujourd'hui en Occident. Toutefois, cette littérature qui, dans sa conception actuelle, relève de la littérature populaire par son souci de plaire et de répondre aux besoins d'un public déterminé, devra évoluer en fonction des changements sociaux qui ne manqueront pas d'affecter les nouvelles générations d'adolescents.

19. Pierre Bruno, "Une enfance, des adolescents", Nous voulons lire!, no 122, Noël 1997, p. 49. 